\title{
Review of: "Comparing quantile regression methods for probabilistic forecasting of NO2 pollution levels"
}

\author{
Ali Jahani ${ }^{1}$ \\ 1 University of Tehran
}

Potential competing interests: The author(s) declared that no potential competing interests exist.

In this manuscript the authors propose an accurate study on development of ten regression models for the predict the distribution of NO2concentrations in a urban location. Authors formulated quantile regression techniques very well and compared the results carefully.

This is a very interesting research work and from my point of view, the idea of this research is very good and fits perfectly within the scope of the journal. In addition, the aims of the paper are consistent with the results and the discussion. The volume of information that the authors have managed is remarkable, and in general the results of the study represent a valuable advance for designing an environmental decision support system tool. I accept this article scientifically. However I suggest some specific comments which are simply revisable by authors.

Specific comments:

- Introduction section need to be expanded with reviewing some recent researches in the field of artificial intelligence application in air quality prediction. I recommend expanding your literature review in order to cover other studies which have been done in the field of artificial neural network modeling instead of focusing on same group of researchers. I suggest these researches to be introduced in Discussion section as the recent development of artificial intelligence models in scientific world

- Except time series data, other influential factors such as traffic, whether, building and barriers and green space factors are missing. May be, you have a reasonable cause for this data but you should compare your methodology with other researches which are using environmental factors to predict air quality of cities. I suggest the results of bellow researches to be reviewed in introduction and discussion:

https://www.sciencedirect.com/science/article/pii/S2212095521000675

https://www.nature.com/articles/s41598-021-81455-6

https://link.springer.com/article/10.1007/s40808-020-00762-5

https://www.researchgate.net/profile/Saba- 
Kalantari/publication/348176798_Forecasting_Ozone_Density_in_Tehran_Air_Using_a_Smart_DataDriven_Approach/links/5ff2a557a6fdccdcb82a80a0/Forecasting-Ozone-Density-in-Tehran-Air-Using-aSmart-Data-Driven-Approach.pdf

- I suggest answer these questions in discussion section: What are the strength and weakness of your research and your suggested method? What could be improved? What are the open questions which you could not answer? 